\title{
Manipulating Task Constraints in Small-Sided Soccer Games: Performance Analysis and Practical Implications
}

\author{
Carlos Humberto Almeida*, António Paulo Ferreira and Anna Volossovitch \\ Sport Expertise Laboratory, Faculty of Human Kinetics, Technical University of Lisbon, Lisbon, Portugal
}

\begin{abstract}
Since an awareness of key task constraints can be extremely beneficial for coaches, the lack of scientific background about the effects of altered game rules/conditions on individual or team performances during soccer practice is surprising. The aim of the present study was to analyze the influence of different small-sided game (SSG) playing rules ("free-form", "two touches" and "four passes to score") on the offensive performance of young soccer players. Eight U-13 male soccer players were divided into two balanced teams. The experimental protocol consisted of three testing sessions separated by one-week intervals. In each session, teams faced each other in the three SSG conditions (3vs.3+goalkeepers) during periods of ten minutes interspersed with five minutes of passive recovery. Simple (i.e. Duration of ball possession, Players involved, Ball Touches, Passes, Shots, and Result of the Offensive Sequence) and composite (i.e. Players involved/Duration, Ball Touches/Duration, Passes/Duration, Ball Touches/Players involved, Passes/Players involved, Passes/Ball Touches, and Goal/Shots) performance indicators were used to characterize the offensive performance of both teams. Results revealed that the factor "playing rule" had a significant effect on simple and composite indicators $(p<0.05)$. It was concluded that manipulating task constraints, such as game rules, can direct practitioners towards intended behaviors, and consequently promote skill acquisition and improve performance in youth soccer. Further research is needed to extend the knowledge about the modification of playing rules in team sports practice.
\end{abstract}

Keywords: Constraints-led approach, performance, rules modifications, small-sided games, technical component, youth soccer.

\section{INTRODUCTION}

Coaches lead the skill development process of players and the improvement of team performance in competitive contexts. The activities proposed in training sessions are essential means for the coach to interact with players, and so accomplish his missions successfully. Particularly in soccer, the "playing form" activities (i.e. small-sided/conditioned games and phase of play exercises) seem to be more relevant to skill acquisition and performance than other types of activities involving physical training and the isolated practice of technical skills [1, 2]. Several researches (e.g. [3-9]) highlight the advantages of using small-sided games (SSGs) to improve physical capacities and technical or tactical skills since the early ages of soccer practice. In this regard, the manipulation of key task constraints, such as pitch dimensions, number of players, rules or conditions, and equipment, is considered a very useful tool for coaches to recreate game situations and contribute to the players' specific skills development [1, 5, 10-12].

Despite this scientific evidence, it seems that coaches are still ignoring the enormous potential of "playing form" activities for fostering skill learning and performance enhancing. For instance, Ford et al. [2] investigated the practice activities employed by 25 coaches working in England with three age categories (Under-9, Under-13, and Under-16 years

*Address correspondence to this author at the Faculty of Human Kinetics, Technical University of Lisbon, Estrada da Costa, 1495-688 Cruz Quebrada, Lisbon, Portugal; Tel.: +351 967990275; Fax: + 351 214144712;

E-mail: carlosalmeida83@hotmail.com old) and three skill levels (elite, sub-elite, and non-elite). These authors pointed out that the percentage of session time spent in conditioned games was $24 \%$ for the elite teams, $11 \%$ for the sub-elite teams, and $9 \%$ for the non-elite teams; considering age categories, the time spent in conditioned games was 15, 18, and $13 \%$ for the U-9, U-13, and U-16 teams, respectively. Even in elite levels, such game situations were underutilized. Furthermore, this seems to be of particular concern in lower age groups, in which young players should be encouraged to participate actively in the learning process through appropriately constrained exploratory practices $[1,11]$.

Although few researches have examined how the modification of playing rules affects physiological and physical demands within SSGs in amateur [13] and young soccer players $[9,14]$, only a couple of studies aimed to investigate the effects of such changes on technical and tactical actions, either in semi-professional [15] or elite professional players [16]. Knowing that the task constraints manipulation can be extremely beneficial for performance improvement, it is remarkable that no study has attempted to evaluate the effects of altered playing rules on sport-specific actions performed during youth soccer practice sessions. Thus, the aim of this study was to analyze the influence of different SSG playing rules ("free-form", "two touches" and "four passes to score") on the offensive performance of young soccer practitioners.

\section{METHODS}

\section{Participants}

Eight U-13 male soccer players (mean $\pm s$; age: $12.8 \pm$ 0.6 years, body mass: $47.14 \pm 14.5 \mathrm{~kg}$, height: $1.56 \pm 0.11 \mathrm{~m}$, training experience: $4.63 \pm 0.74$ years) participated in the 
study. Participants had at least four years of training experience, which consisted of three 90-min sessions per week for nine months of the year. Once a week they represented their club teams competing at the regional level; here, players compete in a seven-a-side championship (i.e., six outfield players plus a goalkeeper per team), which is an official variation of the regular eleven-a-side soccer game (ten outfield players plus a goalkeeper). Parental informed written consent was received prior to experimental sessions and ethics approval was granted by the Scientific Council of the Faculty of Human Kinetics. Participants were distributed into two balanced teams of four players (three outfield players plus a goalkeeper), which were maintained throughout the experiment. Both teams faced each other in all game situations organized.

\section{Experimental Procedures}

After the end of the season, participants completed a protocol consisting of three testing sessions separated by one-week intervals. In each session, teams performed the three game situations during periods of ten minutes interspersed with five minutes of passive recovery. A SSG (3vs.3+GKs) was the basis of the practical procedures; however, it was played under the following three distinct practice rules: (i) "Free-form": players participated in the SSG without additional rules or conditions; (ii) "Two touches": players were allowed to performed a maximum of two consecutive ball touches per individual possession; and (iii) "Four passes to score": teams had to perform at least four consecutive passes to finalize the attack (i.e., to shoot on goal).

All matches were played during the same hours of the day (between 6:00 and 7:00 PM), under warm temperatures $\left(21-25^{\circ} \mathrm{C}\right)$, and with the pitch surface totally dry. Each match was also divided into two 5-min halves, with 1-min interval for midfield exchange. Game duration was based on coach experience, taking into account that each 5-min half do not correspond to the effective playing time due to the stoppages (e.g., fouls, goals, throw-ins, goal kicks, etc.) that normally occur in soccer matches $[4,5]$. Time procedures employed in previous researches concerning SSGs in soccer and futsal were also considered [4, 6, 17-19]. The pitch size of the SSG was $40 \times 30$ meters (length $\mathrm{x}$ width), and it was similar to those used in previous researches, as the pitch ratio per player $\left(150 \mathrm{~m}^{2}\right)[8,20]$. Note that, despite of representing the last line of defense between the opponent and his own team's goal, we agree that the goalkeeper can also play an important role in initiating offensive sequences through effective distribution of the ball [21]; this seems to be especially relevant in modern soccer. For that reason, we calcu- lated the pitch ratio per player including the goalkeepers. The SSGs practice was preceded by a 10-min standardized warm-up encompassing joint mobility exercises and control/passing drills. In order to neutralize the "order effect", game situations were not organized according to the same routine in different sessions (see Table 1). The kick-offs alternated between teams in the beginning of each match and in its second halves. All the official rules of soccer have been implemented apart from the offside rule.

SSGs were conducted in an outdoor soccer field equipped with artificial turf. Two seven-a-side goals (Mondo Portable Goals, Mondo ${ }^{\circledR}$ Corporate, Italy) with the official dimensions of $6 \times 2$ meters (height $\times$ width) and five 4-size soccer balls (Umbro Revolution Storm III Diamon, Umbro ${ }^{\circledR}$ Ltd., England) were used in the experiment, as well as two sets of uniforms (blue and white) in order to differentiate teams. The match time was clocked continuously with a watch (Nike WR0129-001 Triax Speed 50 Super, Nike ${ }^{\circledR}$ Inc., U.S.A.); one collaborator was placed in each touchline of the pitch to reduce the time loss when the ball went out of play. Refereeing was carried out by a neutral collaborator. The practice conditions were explained previously to Session 1 and participants were asked to perform at their best level in order to win the SSGs. No further instruction or feedback about practice was conceded throughout the investigation.

\section{Match Analysis}

Matches were filmed using a digital video camera (Sony DCR-SR77, Sony ${ }^{\circledR}$ Corporation, China) to examine the performance indicators during each SSG condition. The camera was fixed on a tripod (Vivitar V-2200, Vivitar $^{\mathbb{B}}$, U.S.A.) 25 meters behind a seven-a-side goal with an elevation of 10 meters. Images were transferred to a computer via USB and viewed in Windows Media Player (Microsoft ${ }^{\mathbb{B}}$ Corporation, U.S.A.). Afterwards, data were recorded on a Microsoft Office Excel 2007 sheet (Microsoft ${ }^{\mathbb{A}}$ Corporation, U.S.A.) and exported to the SPSS Statistics, version 17.0 (SPSS $^{\circledR}$ Inc., U.S.A.).

The offensive performance was assessed through the characterization of each offensive sequence (i.e., the execution of one or more individual and/or collective tacticaltechnical actions, defined according to criteria of beginning and end of ball possession). The Offensive Sequences Characterization System was the instrument applied for the purpose. Two types of performance indicators were used to characterize the observed offensive sequences: the simple and the composite indicators. Simple indicators derive directly from the behaviors observed through the hand notation analysis system and are simple counts of the game perform-

Table 1. Experimental Procedures

\begin{tabular}{|c|c|c|c|c|c|}
\hline \multirow{6}{*}{$\begin{array}{c}\text { Eight } \\
\text { U-13 Players } \\
\text { Game Format } \\
3 \text { vs. } 3+\text { GKs }\end{array}$} & SESSION 1 & \multirow{6}{*}{ 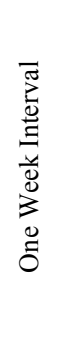 } & SESSION 2 & \multirow{6}{*}{ 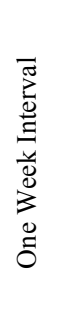 } & SESSION 3 \\
\hline & "Free-form" (2 x 5-min) & & "Two touches" ( 2 x 5-min $)$ & & "Four passes" (2 x 5-min) \\
\hline & $\sqrt{ }$ & & $\sqrt{ }$ & & 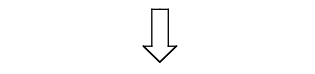 \\
\hline & "Two touches" $(2$ x 5-min $)$ & & "Four passes" (2 x 5-min) & & "Free-form" (2 x 5-min) \\
\hline & $\downarrow$ & & $\downarrow$ & & \\
\hline & "Four passes" $(2 \times 5$-min $)$ & & "Free-form" (2 x 5-min) & & "Two touches" $(2$ x 5 -min $)$ \\
\hline
\end{tabular}


ance: Duration of ball possession, number of Players involved, number of Ball Touches, number of Passes, and number of Shots. The nominal variable Result of the Offensive Sequence is also a simple indicator and assumes one of three forms: i) total success: goal scored; ii) partial success: shot on goal without scoring, the ball hits the goalposts/ crossbar or is saved by the goalkeeper or another player near the goal line; iii) unsuccessful: shot goes wide of the goal, shot intercepted by an opponent, and loss of ball possession.

Moreover, the comparison of performances between teams or different game situations is often facilitated if the performance indicators are expressed as ratios; combining two different performance indicators can provide access to far more meaningful information [22]. So, in order to assist data interpretation, we analyzed composite indicators, which were obtained by dividing two simple indicators: Players involved/Duration of ball possession (rhythm of collective involvement), Ball Touches/Duration of ball possession (rhythm of intervention on the ball), Passes/Duration of ball possession (rhythm of ball transmission between teammates), Ball Touches/Players involved (measurement of individual intervention on the ball), Passes/Players involved (individual contribution to ball transmission), Passes/Ball Touches (playing style adopted by teams), and Goal/Shots (effectiveness of shot). Both types of indicators were then grouped in one of the two levels configured to characterize the offensive sequences: development and finalization.

\section{Reliability}

Some researchers $[6,8]$ have reported the importance of ensuring that observations made during game play are reliable. Hence, prior to the study, an observation protocol was completed to determine the intra-reliability (i.e., the agreement between repeated observations by the same observer) in using the system. The protocol included two analysis sessions spaced at least 7 days apart to prevent that any learning effects influenced the data [6]. In both sessions (test and retest), data corresponding to $20 \%$ of the total images' sample (56 offensive sequences) were observed and notated. The weighted version of kappa statistics [23] was used to evaluate the reliability of both assessments in all simple performance indicators analyzed: Duration of ball possession, number of Players involved, number of Ball Touches, number of Passes, number of Shots, and Result of the Offensive Sequence. There was a very good strength of agreement, since kappa values $(\kappa)$ ranged from 0.81 to 1.0 [24]; these results testify the intra-operator reliability in using the system.

\section{Statistical Analysis}

Data are reported as means $(\bar{x}) \pm$ standard deviations $(s)$, and relative frequencies (\%). After the rejection of the multivariate normality assumption (using Kolmogorov-Smirnov tests for each performance indicator) and the homogeneity of covariance matrices (using Box's M test), non-parametric MANOVAs were applied to evaluate the effects of the factor "playing rule" on simple and composite indicators; for each

Table 2. Performance Indicators Values (Mean $\pm \mathbf{s}$ ) Obtained in Each SSG Playing Rule

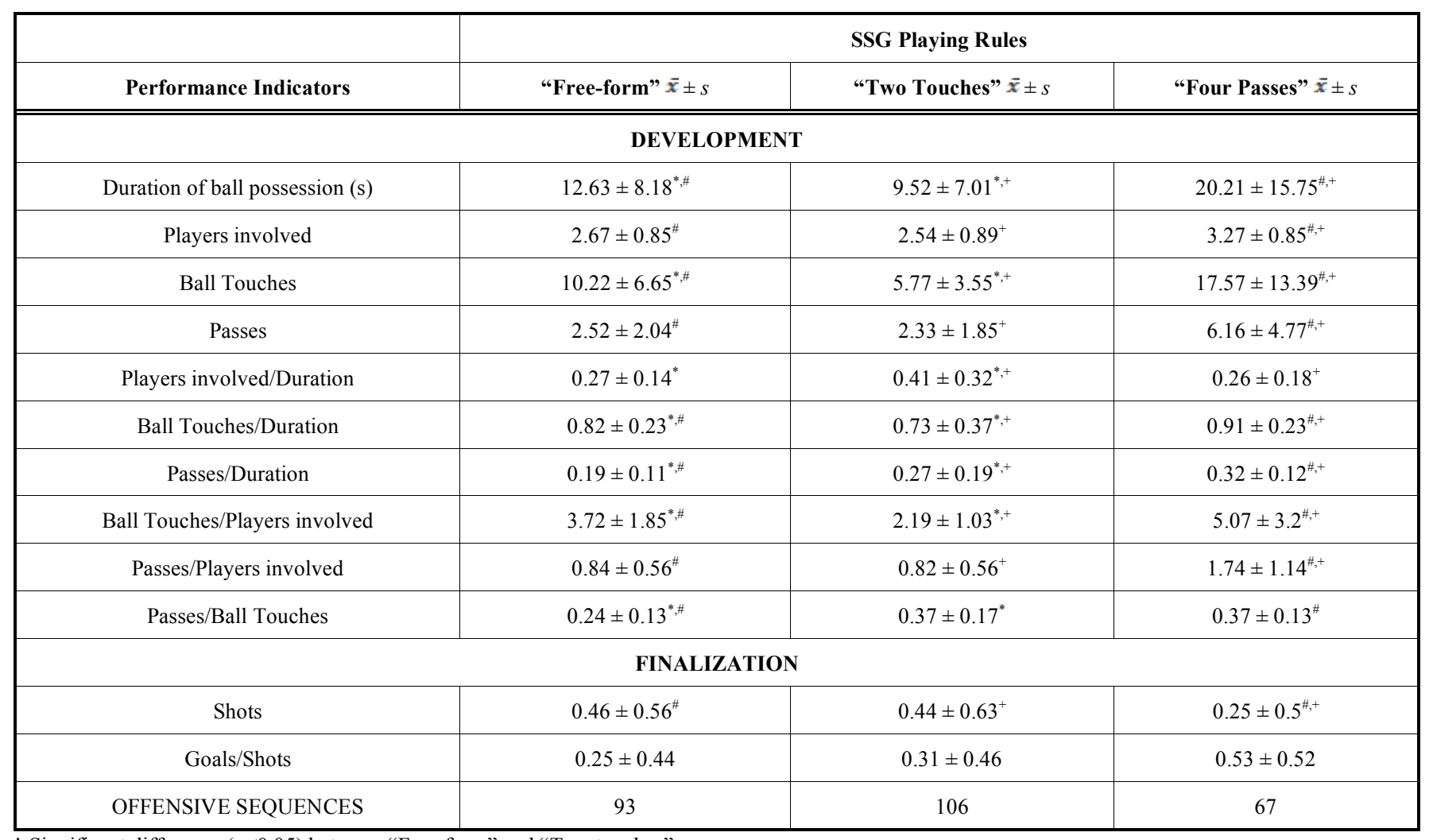

* Significant difference $(\mathrm{p} \leq 0.05)$ between "Free-form" and "Two touches".

\# Significant difference $(\mathrm{p} \leq 0.05)$ between "Free-form" and "Four passes to score".

+ Significant difference $(\mathrm{p} \leq 0.05)$ between "Two touches" and "Four passes to score". 
MANOVA, partials eta squared $\left(\eta_{\mathrm{p}}{ }^{2}\right)$ were calculated as measures of effect size. If significant effects were found, it would be fundamental to identify in which performance indicators the differences occurred. Kruskal-Wallis tests were performed for that purpose, followed by multiple comparisons of mean ranks (LSD post-hoc tests), as described by Maroco [25]. Chi-Square test was used to examine the nominal variable Result of the Offensive Sequence. The level of statistical significance was set at $\mathrm{p} \leq 0.05$.

\section{RESULTS}

Both teams performed a total of 266 offensive sequences in SSGs: 93 in "free-form", 106 with the "two touches" rule, and 67 with the "four passes to score" condition. The descriptive statistics (means \pm standard deviations) of performance indicators that typify the offensive performance produced by participants in each SSG playing rule are shown in Table 2.

Non-parametric MANOVAs revealed that the factor "playing rule" had a significant effect on simple and composite indicators that characterize the offensive sequences $\left(\mathrm{p} \leq 0.001 ; \eta_{\mathrm{p}}{ }^{2}=0.272\right.$, and $\mathrm{p} \leq 0.001 ; \eta_{\mathrm{p}}{ }^{2}=0.416$, respectively). Later, Kruskal-Wallis tests indicated the existence of significant differences $(p \leq 0.05)$ between SSG practice rules in all performance indicators, except for the composite indicator Goals/Shots $(\mathrm{p}>0.05)$.

Finally, post-hoc analyzes confirmed that there were significant differences in Duration of ball possession between all playing rules: "free-form" and "two touches" $(p \leq 0.001)$, "free-play" and "four passes to score" $(p \leq 0.01)$, and "two touches" and "four passes to score" $(\mathrm{p} \leq 0.001)$. The number of Players involved was significantly different between "free-form" and "four passes to score" $(p \leq 0.001)$, and "two touches" and "four passes to score" rules $(\mathrm{p} \leq 0.001)$. The variable Ball Touches also differed significantly between the three SSG conditions: "free-form" and "two touches" ( $p \leq 0.001)$, "free-play" and "four passes to score" $(p \leq 0.001)$, and "two touches" and "four passes to score" $(p \leq 0.001)$. Significant differences were registered between "free-form" and "four passes to score" ( $p \leq 0.001)$, and "two touches" and "four passes to score" $(p \leq 0.001)$ for number of Passes. The number of Shots - concerning the finalization of offensive sequences - was significantly different between "free-form" and "four passes to score" $(\mathrm{p} \leq 0.01)$, and "two touches" and "four passes to score" playing rules $(\mathrm{p} \leq 0.05)$.

With respect to composite indicators, the Players involved/Duration ratio differed significantly between "freeform" and "four passes to score" $(\mathrm{p} \leq 0.001)$, and "two touches" and "four passes to score" $(p \leq 0.001)$. The Ball Touches/Duration, Passes/Duration and Ball Touches/Players involved indicators were significantly different between all playing conditions: "free-form" and "two touches" ( $p \leq 0.001$ for all cases), "free-form" and "four passes to score" $(p \leq 0.05, p \leq 0.001, p \leq 0.05$, respectively), and "two touches" and "four passes to score" ( $\mathrm{p} \leq 0.001$ for all cases). Significant differences were observed between "free-form" and "four passes to score" $(p \leq 0.001)$, and "two touches" and "four passes to score" rules $(p \leq 0.001)$ for the Passes/Players involved ratio. The Passes/Ball Touches indicator differed significantly between "free-form" and "two touches" $(p \leq 0.001)$, and "free-form" and "four passes to score" $(\mathrm{p} \leq 0.001)$.
Data relating to the distribution of the offensive sequences' results in each SSG playing rule are shown in Fig. (1).

Since the number of offensive sequences was different in each practice condition, the relative frequencies provide a more precise view about the results of the offensive sequences. Nevertheless, participants performed more offensive sequences with total success (i.e., goal) in "two touches" SSGs (13), followed by "free-form" (10), and "four passes to score" (8). The number of offensive sequences which ended with partial success (i.e., shot on goal without scoring) was 12 for "free-form", 13 for "two touches", and 3 for "four passes to score" rules. Although young players had concluded a greater number of unsuccessful offensive sequences in "two touches" games (80), followed by "free-form" (71), and "four passes to score" (56), the relative frequencies have revealed the opposite. The statistical procedures demonstrated that the distribution of the offensive sequences' results was independent of the factor "playing rule" $(p>0.05)$, i.e. there were no significant differences between the results of the offensive sequences as a function of different SSGs playing rules.

\section{DISCUSSION}

From a constraints-led perspective applied to team sports, the ability of the coach to manipulate situational constraints in a functional way is fundamental for creating an effective learning environment [11]. According to this standpoint, some authors $[1,10,11]$ have claimed that one of the relevant task constraints that can be manipulated by coaches is the rules/conditions of the game. In fact, our results showed that the SSGs playing rules ("free-form", "two touches", and "four passes to score") have significantly influenced the offensive performance of young participants. In addition, the observed effect sizes on simple and composite performance indicators ranged from medium to large [26], which under-

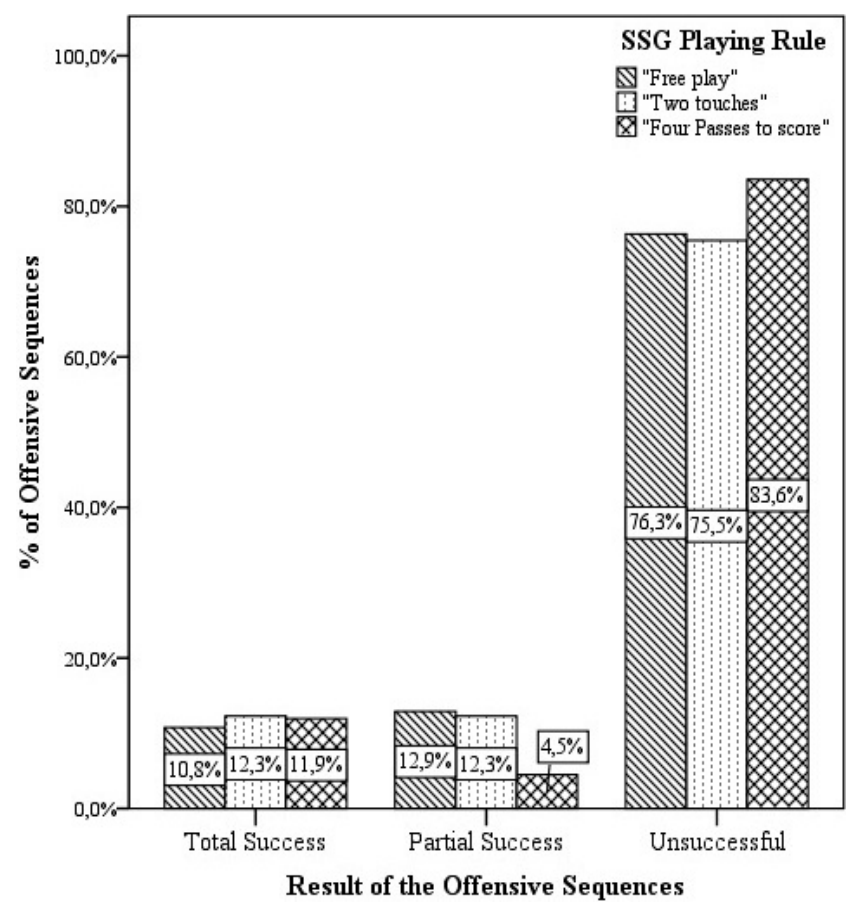

Fig. (1). Result of the offensive sequences (relative frequencies) in each SSG playing rule. 
score the practical importance of manipulating SSGs playing conditions in youth soccer. These evidences support the existing data: the manipulation of key task constraints can lead the learners towards desired sport-specific actions, and so promote skill acquisition and enhance team performance in competitive settings [19].

This research was based on practice contexts that replicate the competitive reality of soccer matches. Such game situations concede to players the opportunity to learn by guided discovery. Ford et al. [2], Button et al. [10], and Davids et al. [11] argue that this kind of "playing form" activities (i.e. small-sided and/or conditioned games) encourage players to participate actively in the learning process, exploring solutions to problems imposed by constraints during practice. For that reason, practitioners should be viewed by coaches as active problem-solvers rather than "empty vessels" or passive recipients of information [1].

Incomprehensively, very few studies have attempted to examine the effects of modified game rules or conditions on skill learning and performance improvement in soccer. The researches of Lemoine et al. [15] and Dellal et al. [16] were a novelty in this issue. Lemoine et al. [15] tried to recreate real game situations using SSGs with the constraint for one team to pass the ball without any time control (i.e., "onetouch playing"); by the analysis of spatial, action and time information, they concluded that this task constraint was efficient, reliable and secure. In turn, Dellal et al. [16] showed that manipulating the number of ball contacts allowed per individual possession influenced, besides other physiological and physical variables, the technical activities of elite soccer players during SSGs. According to Hill-Haas et al. [12], it makes all the sense to extend the still scarce scientific background about the effects of common rules modifications on technical and tactical skills of soccer players. In our view, this is especially relevant in the skill development process during young ages, in which coaches have to pay attention to the fact that youth soccer players do not present the same technical and tactical abilities as the adult or elite players [5].

The present work proved that changing rules is a simple way to have a profound effect on the game performance. Almost all performance indicators were significantly different in dissimilar SSG conditions; the exception was the effectiveness of shot (Goals/Shots). Data confirmed our empirical expectations: "two touches" and "four passes to score" rules are almost opposite conditions, while "freeform" assumes an intermediate position. In this study, the "free-form" condition can be regarded as a control SSG form.

Concerning the development of offensive sequences, the duration of ball possession and the number of ball touches were significantly higher in "four passes to score" games; instead, young players performed shorter offensive sequences and less touches on the ball with the "two touches" rule. The numbers of players involved and passes in the offensive sequences were also significantly superior in SSGs performed with the "four passes to score" condition. According to the values observed in simple performance indicators, "free-form" and "two touches" rules share some features: the mean number of players involved and the mean number of passes were identical. These results pointed out that the "two touches" condition replicates more accurately the competitive nature of the soccer game. Dellal et al. [27] also verified that the number of ball contacts in professional level was close to two touches per possession, and consequently the "free-form" rule would naturally correspond to the "twotouch" type of exercise. Alternatively, the need to perform at least four passes to score affected in a higher degree the style of play adopted by teams; here, the tendency was to attack most of the times against an organized defense, circulating the ball from player to player until the opportunity to shoot on goal emerged.

Lemoine et al. [15] stated that, when players have to perform "one-touch playing" in a momentary configuration of play, it presupposes the adhesion to "an authentic collective project of the game". Despite the "two touches" SSGs being identical to the "one-touch playing", our results do not reinforce that supposition, when compared to the other SSG conditions. Since the "four passes to score" rule provided a greater collective involvement of players in the analyzed matches, it seems to be a better training stimulus to develop collective processes of play in the attacking phase. Most of all, coaches should understand that, besides the development of physical performance (e.g., maximal aerobic capacity and ability to repeated sprints), young players need to improve technical and tactical skills [5]. Therefore, the use of "two touches" and "four passes to score" conditions seems to represent high-quality alternatives to optimize these components in youth soccer.

Regarding the composite indicators, the rhythm of collective involvement in the offensive sequences (Players involved/Duration) was considerably greater when teams played with the "two touches" rule; in "free-form" and "four passes to score" conditions similar mean values were obtained. Dellal et al. [27] observed that the modern high-level soccer demands playing quickly in offensive situations and to put pressure on the opponent as quickly as possible in defensive situations. In this sense, the "two touches on the ball" constraint requires that the attackers play faster in order to take an advantage or to disrupt the opponent's defense [15]. Moreover, as defenders know that the attackers are not able to dribble them, due to such task constraint, the defensive pressure on the player with ball possession intensifies. As a consequence, the rhythm of collective involvement during offensive sequences increased. Nonetheless, participants presented significantly greater rhythms of ball intervention (Ball Touches/Duration) and ball transmission between teammates (Passes/Duration), as well as significantly higher values in the individual relation with the ball (Ball Touches/Players involved) and individual contribution to ball circulation (Passes/Players involved) playing with the rule of "four passes to score". Participants have more time and space - because the defensive pressure is reduced - to keep the ball possession and execute more ball touches and passes in this type of "possession games". Besides the need to perform at least four consecutive passes, the restriction to carry out a certain number of touches on the ball also accelerates the rhythm of offensive communication between teammates. This explanation elucidates why "four passes to score", "two touches", and "free-form" rules differed significantly in this particular performance indicator. 
The ratio Passes/Ball Touches was similar in "two touches" and "four passes to score" conditions and shows a tendency to develop predominantly offensive sequences through collective actions. On the contrary, the SSGs with the "free-form" rule differed significantly from the other two practice conditions; in this case, participants sought to produce offensive sequences according to a more individualistic approach, which corroborates the data obtained by Dellal et al. [16]. They observed that the number of duels and the duels per minute of play were superior in "free-form" compared to the "two touches" rule for the 4 vs. 4 format. The simple SSG offers players the possibility to retain ball possession without further constraints, which leads them to choose not only to pass the ball in advantageous circumstances for their team, but also to explore 1 vs. 1 duels in appropriate moments.

The finalization level of analysis demonstrated that players performed a significant greater number of shots per offensive sequence in SSGs with "free-form" and "two touches" conditions, when comparing to the "four passes to score" rule. This fact reveals that participants shot more and scored more goals using faster offensive methods. Carling et al. [21], and Hughes and Churchill [28] found that most of the goals obtained in international competitions resulted from short passing sequences and ball possessions with shorter duration. These authors pointed out the effectiveness of fastest attacks in the rupture of opposing defensive organization. In spite of that, the effectiveness of shot was higher when teams were playing with the "four passes to score" rule than in the other SSGs. This result indicates that, when the team adopts a "possession play" style, players tend to find better chances to shoot on goal. Although young players have concluded more unsuccessful attacks in "two-touch" playing, they reached a higher percentage of unsuccessful attacks during the "four passes to score" constraint. Results have shown that the number of individual mistakes tends to be clearly superior when the behavior of the player with the ball is constrained. Dellal et al. [16] reported that the number of duels and the percentage of successful passes decreased from "free-form" to "one touch" game conditions (except for 3 vs. 3), whereas the number of balls lost increased. This might be due to the difficulty of finding effective solutions to tactical problems that emerged in game contexts with increased defensive pressure. So, when the team plays against an organized defense, as occurs with the "four passes to score" rule, the possibility to explore free spaces near the goal is reduced, which affects the number of the team's successful offensive sequences.

Furthermore, the standard deviations presented by both teams across all playing rules suggest much more variability in the development of offensive sequences under the "four passes to score" constraint. Data indicate that different task constraints impose restrictions at distinct levels on playing solutions. "Two touches" condition seems to inflict more restrictions in the development of offensive sequences, while the "four passes to score" rule decreases the opportunities for players to shoot on goal. In this paper, we haven't directly examined the effects of different playing rules on skill acquisition; that is a limitation to acknowledge. Perhaps, it would be interesting to investigate how such game constraints influence the acquisition of sport-specific skills in youth soccer players.
Our research supports that manipulating game rules or conditions is a selective tool that allows enhancing the quality of the coaching tasks. A constraint such as playing with two touches forces players to "read the game", make emergent decisions, and perform faster. The imposition to complete a certain number of consecutive passes to finalize the attack enables practitioners to act better as a team, circulating the ball accurately and finding solutions to penetrate the opposing defensive system. However, coaches must be aware that adding task constraints creates artificial situations, and if overused, can promote bad habits [29]. For instance, the "two touches" playing rule does not provide to youngsters the best circumstances to develop the dribbling actions useful in 1 vs. 1 situations. As a result, the modification of game rules should be carefully pondered and applied for specific purposes, when planning youth soccer training sessions.

\section{CONCLUSION}

The present research revealed that the playing rules, imposed to young players in a SSG, influenced their offensive performance. Such evidence confirms that manipulating key task constraints can direct practitioners towards an intended outcome, thus promoting skill acquisition and performance improvement in team sports. Whereas the "two touches" condition required from players to act faster, the "four passes to score" rule fosters the offensive communication among team members. In SSGs with the "free-form" rule, participants adopted a more individualistic approach. Data also demonstrated that "two touches" and "free-form" conditions enable players to produce faster offensive sequences, and therefore to shoot more on goal and score more goals.

Coaches should be widely encouraged to vary practice contexts by manipulating constraints such as game rules or conditions. Despite that, the use and frequency of task constraints should be well pondered, so that skill acquisition and performance improvement can be effective over the years of soccer training. Further researches should extend this work by analyzing these or other situational constraints (e.g., scoring off a cross only, modifying the number/position of goals, restricting areas to shoot on goal, etc.), with participants from other age categories. Additionally, by including other variables that capture the dynamics of players' displacements during match-play, future researches can provide valuable information about the interpersonal coordination among players inside the teams and between teams in an opposing relationship [18].

\section{CONFLICT OF INTEREST}

The authors confirm that this article content has no conflicts of interest.

\section{ACKNOWLEDGEMENT}

Declared none.

\section{REFERENCES}

[1] Williams AM, Hodges NJ. Practice, instruction and skill acquisition in soccer: challenging tradition. J Sports Sci 2005; 23(6): 63750 . 
[2] Ford PR, Yates I, Williams AM. An analysis of practice activities and instructional behaviours used by youth soccer coaches during practice: exploring the link between science and application. J Sports Sci 2010; 28(5): 483-95.

[3] Capranica L, Tessitore A, Guidetti L, Figura F. Heart rate and match analysis in pre-pubescent soccer players. J Sports Sci 2001; 19: 379-84.

[4] Casamichana D, Castellano J. Time-motion, heart-rate, perceptual and motor behaviour demands in small-sided soccer games: effects of pitch size. J Sports Sci 2010; 28(14): 1615-23.

[5] Dellal A, Jannault R, Lopez-Segovia M, Pialoux V. Influence of numbers of players in the heart rate responses of youth soccer players within 2 vs. 2,3 vs. 3 and 4 vs. 4 small-sided games. J Hum Kinet 2011; 28: 107-14.

[6] Jones S, Drust B. Physiological and technical demands of $4 \mathrm{v} 4$ and $8 v 8$ games in elite youth soccer players. Kinesiology 2007; 39(2): $150-6$

[7] Katis A, Kellis E. Effects of small-sided games on physical conditioning and performance in youth soccer players. J Sports Sci Med 2009; 8: 374-80.

[8] Kelly DM, Drust B. The effect of pitch dimensions on heart rate responses and technical demands of small-sided soccer games in elite players. J Sci Med Sport 2009; 12: 475-9.

[9] Hill-Haas SV, Coutts AJ, Dawson BT, Rowsell GJ. Time-motion characteristics and physiological responses of small-sided games in elite youth players: the influence of player number and rule changes. J Strength Cond Res 2010; 24(8): 2149-56.

[10] Button C, Smith J, Pepping GJ. The influential role of task constraints in acquiring football skills. In: Reilly T, Cabri J, Araújo D, Eds. Science and football V: the proceedings of the fifth world congress on science and football; 2003: London, UK: Routledge, Taylor \& Francis Group 2005; pp. 500-8.

[11] Davids K, Araújo D, Shuttleworth R. Applications of dynamical systems theory to football. In: Reilly T, Cabri J, Araújo D, Eds. Science and football V: the proceedings of the fifth world congress on science and football; 2003: London, UK: Routledge, Taylor \& Francis Group 2005; pp. 556-69.

[12] Hill-Haas SV, Dawson B, Impellizzeri FM, Coutts A. Physiology of small-sided games training in football: a systematic review. Sports Med 2011; 41(3): 199-220.

[13] Aroso J, Rebelo A, Gomes-Pereira J. Physiological impact of selected game-related exercises. J Sports Sci 2004; 22: 522.

[14] Sampaio J, Garcia G, Maçãs V, Ibanez J, Abrantes C, Caixinha P. Heart rate and perceptual responses to $2 \times 2$ and $3 \times 3$ small-sided youth soccer games. J Sports Sci Med 2007; 6 (Suppl. 10): 121-2.
[15] Lemoine A, Jullien H, Ahmaidi S. Technical and tactical analysis of one-touch playing in soccer: study of the production of information. Int J Perform Anal Sport 2005; 5(1): 83-103.

[16] Dellal A, Chamari K, Owen AL, Wong DP, Lago-Penas C, HillHaas S. Influence of technical instructions on the physiological and physical demands of small-sided soccer games. Eur J Sport Sci 2011; 11(5): 341-6.

[17] Duarte R, Batalha N, Folgado H, Sampaio J. Effects of exercise duration and number of players in heart rate responses and technical skills during futsal small-sided games. Open Sports Sci J 2009; 2: 37-41.

[18] Frencken W, Lemmink K, Delleman N, Visscher C. Oscillations of centroid position and surface area of soccer teams in small-sided games. Eur J Sport Sci 2011; 11(4): 215-23.

[19] Duarte R, Araújo D, Fernandes O, et al. Effects of different practice task constraints on fluctuations of player heart rate in smallsided football games. Open Sports Sci J 2010; 3: 13-5.

[20] Hill-Haas SV, Dawson BT, Coutts AJ, Rowsell GJ. Physiological responses and time-motion characteristics of various small-sided soccer games in youth players. J Sports Sci 2009; 27(1): 1-8.

[21] Carling C, Williams AM, Reilly T. Handbook of soccer match analysis: a systematic approach to improving performance. London, UK: Routledge, Taylor \& Francis Group 2005.

[22] Hughes MD, Bartlett RM. The use of performance indicators in performance analysis. J Sports Sci 2002; 20: 739-54.

[23] Robinson G, O’Donoghue P. A weighted kappa statistic for reliability testing in performance analysis of sport. Int J Perform Anal Sport 2007; 7(1): 12-9.

[24] O'Donoghue P. Research methods for sports performance analysis. London, UK: Routledge, Taylor \& Francis Group 2010.

[25] Maroco J. Análise estatística - Com utilização do SPSS. $3^{\text {rd }}$ ed. Lisboa: Edições Sílabo, Lda. 2010.

[26] Cohen J. A power primer. Psychol Bull 1992; 112(1): 155-9.

[27] Dellal A, Chamari K, Wong DP, et al. Comparison of physical and technical performance in European soccer match-play: FA premier league and la liga. Eur J Sport Sci 2011; 11(1): 51-9.

[28] Hughes M, Churchill S. Attacking profiles of successful and unsuccessful teams in Copa America 2001. In: Reilly T, Cabri J, Araújo D, Eds. Science and football V: The proceedings of the fifth world congress on science and football; 2003: London, UK: Routledge, Taylor \& Francis Group 2005; pp. 222-8

[29] Paul L. Playing better soccer is more fun: a comprehensive guide to the small-sided games coaching model. Springfield, VA: Accotink Press 2005 .

(C) Almeida et al.; Licensee Bentham Open.

This is an open access article licensed under the terms of the Creative Commons Attribution Non-Commercial License (http://creativecommons.org/licenses/ by-nc/3.0/) which permits unrestricted, non-commercial use, distribution and reproduction in any medium, provided the work is properly cited. 